\title{
High Frequency Distributed Optical Fibre Dynamic Strain Sensing: A Review
}

\section{Introduction}

The concept of using an optical fibre to map dynamic perturbations along the sensing fibre was first proposed by Taylor et al. (Taylor 1993) in 1993 and later demonstrated by his team in 2005 (Juarez 2005). The proposed sensor was only capable of detecting the dynamic perturbations along the sensing fibre by monitoring the changes in the pattern of the backscattered coherent Rayleigh noise (CRN). The volume of research since then has proliferated and has mainly been focused on developing distributed optical fibre dynamic strain sensors capable of fully quantifying the characteristics of perturbations. The main driving force behind the expansion of this field has been the growing demand in areas such as the oil and gas industries, geophysical sciences, and structural health monitoring.

Distributed optical fibre dynamic strain sensors can be broadly divided into two categories: (1) sensing systems based on Brillouin scattering and (2) sensing systems based on Rayleigh scattering. Brillouinbased distributed optical fibre sensors (DOFS) measure the absolute strain and temperature along the fibre using the Brillouin frequency shift (Bernini 2009 and Peled 2011). Although this class of sensors have a higher strain range, their applications as a dynamic strain sensor are limited to short sensing ranges due to the negative trade-off between the frequency and sensing range (Masoudi 2016).

Rayleigh-based Distributed vibration sensors (DVS), on the other hand, have demonstrated a longer sensing range with higher strain resolution. This class of sensors are more suitable in areas such as geophysical sciences and structural health monitoring where the sensor is required to monitor small strain variations over large distances. DVS based on Rayleigh scattering do not provide the absolute strain, but rather the magnitude of the strain fluctuations. The focus of this article is to study this class of sensors.

\section{Principle}

The underlying principle of Rayleigh-based DVS is based on phase optical time domain reflectometry $(\varphi-O T D R)$ sensing technique which uses the phase of the Rayleigh backscattered signal to measure vibration. For any given section of the fibre, the phase-difference between the backscattered light from the two ends of that section, $\Delta \phi$, is a function of the length of that section, $L$ (figure 1 ). For any unperturbed section of the sensing fibre, the length and, consequently, the phase-difference remains

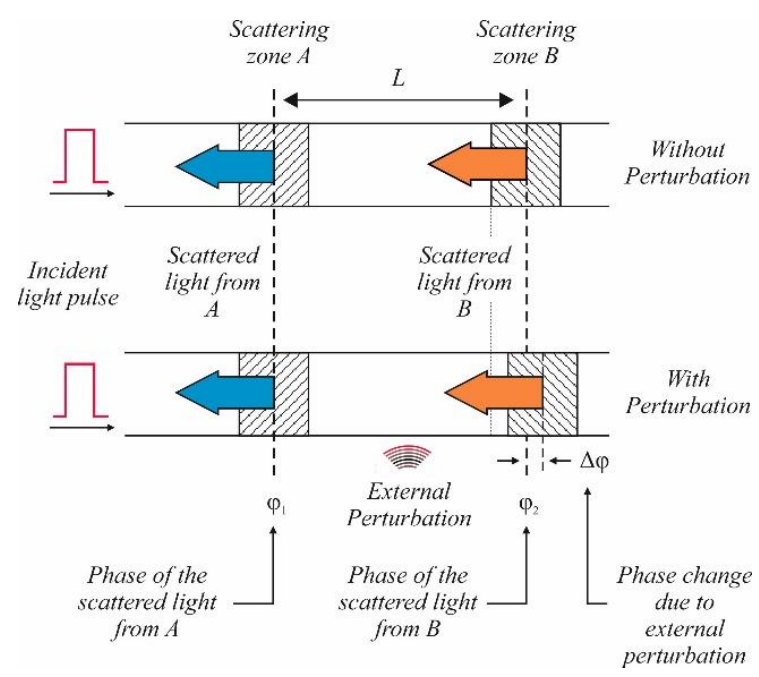

Figure 1 Principle of the dynamic strain sensor using $\varphi$-OTDR. This figure represents the behaviour of two groups of scatterers inside the sensing fibre before and after external perturbation. 
unchanged. Any perturbation which induces a strain $\varepsilon$ on any section of the fibre will alter the phasedifference. The changes in the phase-difference as a function of length is given by (Hocker 1979):

$$
\Delta \phi=\varepsilon L\left[\beta-\frac{1}{2} \beta n^{2}\left[(1-\mu) p_{12}-\mu p_{11}\right]\right]
$$

where $\beta$ is the propagation constant of light in the fibre, $n$ is the refractive index of the fibre, $\mu$ is the Poisson's ratio, and $P_{11}$ and $P_{12}$ are strain-optic coefficients. By monitoring the variation in the phase difference of the backscattered light from any two sections along the sensing fibre, the strain variation of that section can be measured.

\section{$\varphi$-OTDR Sensing Techniques}

The sensing mechanism of $\varphi$-OTDR DVS falls into three categories:

a) Dual-pulse technique: This technique is based on sending a pair of pulses temporally separated by $\Delta T$ seconds to interrogate the fibre (figure 2(a)). The temporal separation between the pulses results in the backscattered light from the first pulse at location $x_{1}$ to be mixed with the backscattered light from the second pulse at location $x_{2}$, with the spatial separation between $x_{1}$ and $x_{2}$ is given by $\Delta X=\Delta T . c / 2 n$.

This sensing technique was first proposed by Dakin and Lamb (Dakin 1990) in which they used an acousto-optic modulator (AOM) to simultaneously modulate the $\mathrm{CW}$ light to form the probe pulses and shift their frequencies. The combination of the two backscattered traces from the two pulses results in a signal at the photodetector with a beat frequency of $\Delta f=\left|f_{1}-f_{2}\right|$. Dakin and Lamb showed that the phase of the beat signal for any given section of the fibre has a linear relationship with the strain of that section. Although, in theory, the proposed concept could provide the phase information along the sensing fibre, in practice, the setup requires an unrealistic control over the timing of the pulse pair and,

a)

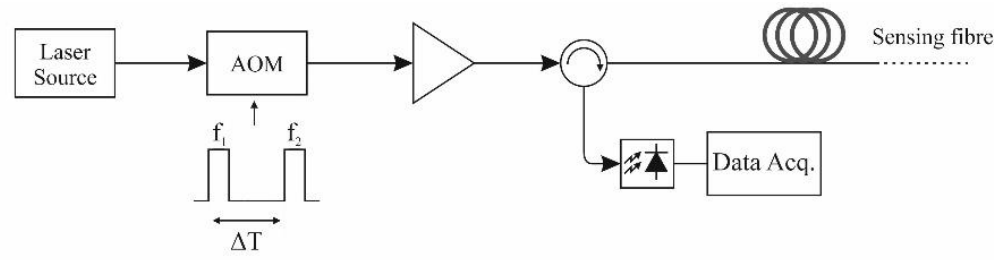

b)

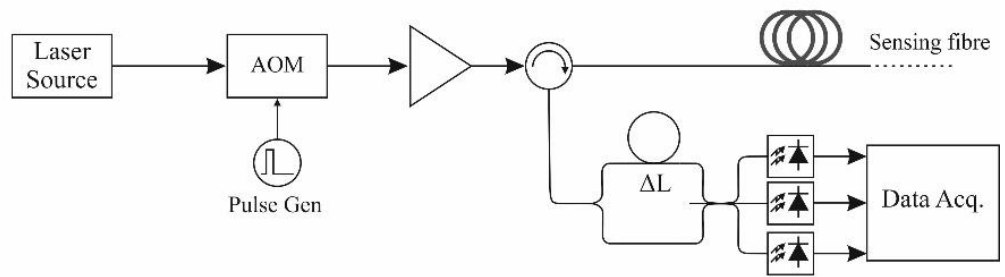

c)

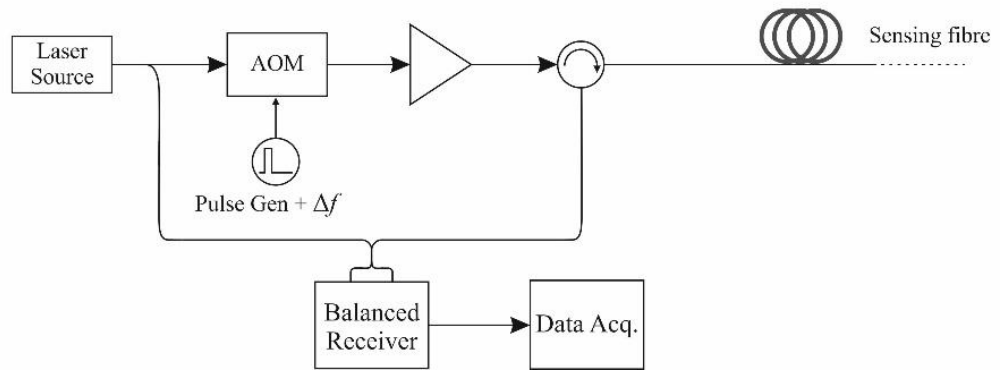

Figure 2 The experimental setups of three DVS, the operations of which are based on $\varphi$-OTDR. 
in addition, it may suffer from signal fading. In 2014, Alekseev et al. (Alekseev 2014) experimentally demonstrated a modified version of this concept by using a semiconductor optical amplifier to generate 100ns pulses while controlling the relative phase of pulse pair using an electro-optic modulator (EOM). The experimental results showed that this sensing technique is capable of measuring $230 \mathrm{~Hz}$ periodic perturbations along a $2 \mathrm{~km}$ long sensing fibre with a spatial resolution of $5 \mathrm{~m}$ and a strain resolution of $10 n \varepsilon$.

b) Interferometry technique: In the year 2000, Posey et al. (Posey 2000) demonstrated an alternative measurement technique in which the relative phase of the backscattered light between two separate sections was measured using an imbalanced Mach-Zehnder interferometer (MZI) (figure 2(b)). In this technique, the backscattered light from a single pulse is inserted into an imbalanced MZI with two unequal arms to provide two similar traces with temporal shift of $\Delta T=\Delta L . n / c$, where $\Delta L$ is the path imbalance of the MZI. To avoid signal fading, the signals from two arms of the IMZI are mixed in a $3 \times 3$ coupler to provide three waves with a nominal phase-shift of $120^{\circ}$ between them.

A modified version of Posey's proposal was later realized by Masoudi et al. (Masoudi 2013) where instead of measuring the vibrations along the sensing fibre one point at a time, the entire length of the sensing fibre was mapped simultaneously. Using this technique, Masoudi et al. demonstrated a $1 \mathrm{~km}$ long distributed vibration sensor with a strain and frequency range of $2 \mu \varepsilon$ and $4 \mathrm{kHz}$, respectively. The spatial resolution of the sensor was measured to be $1 \mathrm{~m}$. Fang et al. (Fang 2015) demonstrated an alternative implementation of this technique using an imbalanced Michelson interferometer instead of MZI. More recently, Masoudi and Newson (Masoudi 2017) have proposed a modified the experimental setup and signal processing procedure to improve the sensitivity and spatial resolution of the DVS to $40 \mathrm{n} \varepsilon$ and $50 \mathrm{~cm}$, respectively.

c) Electrical-domain technique: This technique which first disclosed by Hartog and Kader (Hartog 2012) is based on converting the data from the optical domain to the electrical domain before measuring the phase difference between two sections of the sensing fibre in the electrical domain. In this approach, an acousto-optic modulator (AOM) is used to generate optical pulses with a frequency shift of $\Delta f$, relative to the light source. The backscattered light from the fibre is mixed with the light source at a balanced photodetector to generate a beat signal which retains both the amplitude and phase information. The phase information along the fibre is then measured in the electrical domain using a phase detection circuit.

This technique was validated by Tu et al. (Tu 2015) and later by Yang et al. (Yang 2016). The experimental results published by Yang et al. showed that this sensing technique is capable of spatially resolving $1 \mathrm{kHz}$ dynamic strains along $30 \mathrm{~km}$ of sensing fibre with a spatial and strain resolution of $10 \mathrm{~m}$ and $50 n \varepsilon$, respectively.

\section{Discussion \& Conclusion}

So far, three different sensing techniques have exhibited the capability of measuring high frequency dynamic perturbations over long sensing ranges. All three techniques use the changes in the phase of the backscattered Rayleigh signal to measure dynamic strains along the sensing fibre. The experimental results show that the strain resolution of all three techniques are within the same order of magnitude $(10 \mathrm{n} \varepsilon \sim 50 \mathrm{n} \varepsilon)$ albeit over different gauge length.

The main limitation of the three sensing technique is the linearity of their responses. Since the measured strain at each section of the sensing fibre is measured using the phase-difference between zones at two ends of that section, the intrinsic phases of those zones play an important role in the linearity of the response. If the distribution of the inhomogeneities of those zones are not affected by the perturbation (i.e. local perturbation), the strain between the two zones would have a linear relationship with the phase. Any perturbation where the entire fibre is affected alters the intrinsic phase of the zones where the phase are sensed which, in turn, results in non-linearity in the measured phase. 


\section{Acknowledgment}

This work is funded by EPSRC UK grants EP/N00437X/1.

\section{References}

Alekseev, A.E., Vdovenko, V.S., Gorshkov, B.G., Potapov, V.T. and Simikin, D.E. [2014] A phasesensitive optical time-domain reflectometer with dual-pulse phase modulated probe signal. Laser Physics, 24 (11), 115106.

Bernini, R., Minardo, A. and Zeni, L. [2009] Dynamic strain measurement in optical fibers by stimulated Brillouin scattering. Optics Letters, 34(17), 2613-5.

Dakin, J.P. and Lamb, C. [1990] Distributed fibre optic sensor system. GB patent 2222247 A.

Fang, G., Xu, T., Feng, S. and Li, F. [2015] Phase-sensitive optical time domain reflectometer based on phase-generated carrier algorithm. Journal of Lightwave Technology, 33 (13), 2811-6.

Hartog A.H. and Kader K. [2012] Distributed fiber optic sensor system with improved linearity. U.S. patent No. 2012/0067118 A1.

Hocker, G.B. [1979] Fiber-optic sensing of pressure and temperature. Applied Optics, 18(9), 1445-8.

Juarez, J.C., Maier, E.W., Choi, K.N. and Taylor, H.F. [2005] Distributed fiber-optic intrusion sensor system. Journal of Lightwave Technology, 23 (6), 2081-7.

Masoudi, A., Belal, M. and Newson, T.P. [2013] A distributed optical fibre dynamic strain sensor based on phase-OTDR. Measurement Science and Technology, 24 (8), 085204.

Masoudi, A. and Newson, T.P. [2016] Contributed Review: Distributed optical fibre dynamic strain sensing. Review of Scientific Instrument, 87 (1), 011501.

Masoudi, A. and Newson, T.P. [2017] High spatial resolution distributed optical fibre dynamic strain sensor with enhanced frequency and strain resolution. Optics Letters, 42 (1), accepted manuscript.

Peled, Y., Motil, A., Yaron, L. and Tur, M. [2011] Slope-assisted fast distributed sensing in optical fibers with arbitrary Brillouin profile. Optics Express, 19 (21), 19845-54.

Posey, R.J., Johnson, G.A. and Vohra, S.T. [2000] Strain sensing based on coherent Rayleigh scattering in an optical fiber. Electronics Letters, 36 (20), 1688-9.

Taylor, H.F. and Lee, C.E. [1993], Apparatus and method for fiber optic intrusion sensing. US patent US5194847 A.

Tu, G., Zhang, X., Zhang, Y., Zhu, F., Xia, L. and Nakarmi, B. [2015] The development of an $\varphi-O T D R$ system for quantitative vibration measurement. IEEE Photonics Technology Letters, 27 (12), 1349-52.

Wang, C., Wang, C., Shang, Y., Liu, X. and Peng, G. [2015] Distributed acoustic mapping based on interferometry of phase optical time-domain reflectometry. Optics Communications, 346, 172-7.

Yang, G., Fan, X., Wang, S., Wang, B., Liu, Q. and He, Z. [2016] Long-Range distributed vibration sensing based on phase extraction from phase-sensitive OTDR. IEEE Photonics Journal, 8 (3), 6802412 . 